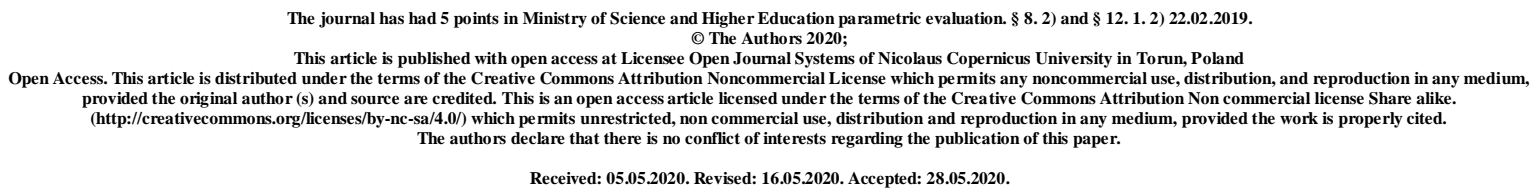

\title{
Acetazolamide in prevention of altitude disease
}

\author{
${ }^{1}$ Pawel Choroszewicz, ${ }^{1}$ Anna Maria Dobosiewicz, ${ }^{2}$ Nataliia Badiuk
}

\author{
${ }^{1}$ Scientific Circle of Exercise Physiology at Department of Hygiene, Epidemiology and \\ Ergonomics. Division of Ergonomics and Exercise Physiology, Nicolaus Copernicus \\ Univeristy in Toruń, Collegium Medicum in Bydgoszcz, Poland \\ ${ }^{2}$ State Enterprise Ukrainian Research Institute for Medicine of Transport of Ukrainian \\ Ministry of Health Care", Odesa, Ukraine
}

\begin{abstract}
Mountain climbing is associated with many risky, life-threatening situations. One of the most common attacking diseases at altitudes above $2500 \mathrm{~m}$ is altitude disease (HAI). This disease consists of: acute alpine disease, cerebral oedema and pulmonary oedema at altitude.

The best way to prevent altitude diseases is by means of alcomatisation and slow increase in altitude. Climbers should follow the principle "climb high, sleep low". Sometimes, however, time or inability to descend do not allow for acclimatization. In such conditions, it is necessary to use pharmaceuticals that prevent the occurrence of an illness of altitude.

The following article discusses research on the most commonly used drug in the prevention of acute mountain disease - acetazolamide. This drug was first used in the 60s of the XX century, demonstrating its effectiveness in the prevention of mountain disease. Since then, it has been the subject of many studies.
\end{abstract}

Key words: HAPE; HACE; AMS; acetazolamide 


\section{Introduction}

High Altitude Disease (HAI) is a syndrome that can attack after exceeding $2500 \mathrm{~m}$ during mountain climbing [1]. It consists of three different syndromes:

- AMS- acute mountain climbing disease

- HACE-height cerebral oedema

- HAPE- hepatic pulmonary oedema [7].

The disease is caused by hypoxia resulting from high altitude and hypoxia.

AMS most commonly occurs above $2500 \mathrm{~m}$ above sea level; the risk of HAPE increases above $3000 \mathrm{~m}$ and HACE above $4000 \mathrm{~m}$ [10].

The mechanism of AMS formation is not fully understood. The researchers assume that hypoxaemia leads to increased cerebral perfusion and increased capillary pressure. If the permeability of the blood-brain barrier is increased simultaneously with the phenomena mentioned, cerebral oedema may develop. Attention is also drawn to the diastole of smooth muscles in the walls of cerebral vessels and disorders of cerebral autoregulation. Increased amount of cerebrospinal fluid may also be the underlying mechanism of AMS formation. It has been found that individuals with less fluid are less likely to develop cerebral oedema [16].

The symptoms of AMS usually appear 6-12 $\mathrm{h}$ after exposure to the factor. The highest intensity of symptoms occurs 2-3 days after the disease. The return to proper functioning of the body is most common on day 4-5 [1, 7, 23]. The symptoms of AMS differ from the disease [23]. In mild forms of the disease, weakness, fatigue, anorexia, nausea and headache are observed [1, 14, 23]. The acute form of AMS causes increased occurrence of the above mentioned symptoms and is characterized by: stinginess, dyspnea and vomiting [14, 23].

According to the Lake Louise Consensus Group, which was established during the Intenational Hypoxia Symposium in Canada in 1991, headache and one or more of the following symptoms must be present for a diagnosis of AMS:

- Gastrointestinal complaints such as: lack of appetite, nausea, vomiting

- Suffocation

- Dizziness

- Feeling tired $[16,17]$.

The best way to prevent AMS is acclimatization. It is recommended to increase the height by $300-500 \mathrm{~m}$ daily $[1,6]$. In situations when acclimatization is not possible, prevention with pharmacological agents is recommended [8]. In this case, acetazolamide in the dose: 125-250 mg twice daily or dexamethasone in the dose: 4mg 2-3 times daily [1]. However, 
dexamethasone is not used first. It is used only if the climber is allergic to acetazolamide or has to be quickly transported to higher parts of mountains [8].

If the disease occurs, treatment should be implemented immediately. Mild AMS can be treated with non-steroidal anti-inflammatory and antiemetic drugs without the necessity to go to the lower parts of mountains. If no improvement or progression of symptoms is observed, it should go lower [10].

The Lake Louise Assessment Questionnaire is most commonly used to assess the severity of AMS. However, not only the result of the questionnaire should be suggested, but the patient should also be examined. The questionnaire allows us to assess the extent to which the following symptoms are present: headache, gastrointestinal disorders, dizziness/unbalance, fatigue. The listed symptoms are assigned points from 0 to 3 . The sum of points greater than 3 may indicate the occurrence of AMS [16].

A longer duration of acute mountain disease may lead to elevated pulmonary oedema (HAPE) and elevated brain oedema (HACE) [1, 10]. If vomiting is repeated and the headache does not go away during AMS after the analgesics used, it may mean that AMS is now progressing to HACE [6].

Brain oedema develops about $12 \mathrm{~h}$ after exposure above $3500 \mathrm{~m}$. The mortality rate is about $60 \%$ when a coma occurs [23].

Both HACE are hallucinations, fuzzy speech, ataxia, convulsions, and in the final phase a coma occurs $[1,23]$. Ataxia is a symptom of the early stage of brain oedema. [23]

According to the Lake Louis Consensus, elevated cerebral swelling is found if: there is a consciousness disorder with or without ataxia (for people who previously had AMS symptoms). Simultaneous occurrence of consciousness disorders and ataxia (in the case of individuals who have not previously experienced symptoms of AMS) [16].

If HACE symptoms are observed during climbing, they should immediately descend below [1]. HACE is treated with oxygen therapy, a game-bag, change of place on the lower part of mountains. In pharmacotherapy, dexamethasone is initially put on dexamethasone in the dose of $8 \mathrm{mg}$, then $4 \mathrm{mg} 4$ times a day [1, 11,23]. In cases of seizures, 10-20 mg of relanum are administered. Once the coma develops, it is important for airways to be clear; mannitol and fursemide are used [23].

Under the influence of increasing resistance in lung vessels, the pressure in the pulmonary artery increases, which in turn leads to pulmonary oedema (HAPE). This is the commonest cause of death at mountain altitudes.

Pulmonary oedema may contribute to the occurrence of HAPE: 
- Rapid altitude change

- Low temperature

- High salt content

- Intense effort

- Sleeping pills

Symptoms of elevated pulmonary oedema are: dyspnea occurring during exercise and at a later stage also at rest, dry coughing with discharge spitting out, also AMS symptoms intensify. In the next stage of the disease, body temperature increases and tachycardia occurs $[14,23]$. The treatment of pulmonary oedema includes oxygen therapy, effort limitation and transport of patients about 500-1000 m lower. Moreover, a gamma bag, which is a kind of hyperbaric chamber, is used. Thus, the patient is able to breathe under increased partial pressure [23]. Moreover, calcium channel blockers and PDE-5 inhibitors are effective in the treatment of acute pulmonary oedema [22].

In prevention of HAPE, inhibitors of nifedipine, salmeterol and phosphodiesterase are used [7].

As established in Lake Louis Consesus, to determine elevated pulmonary oedema at least 2 symptoms should be observed:

- Cough

- Resting breathlessness

- Weakening/minority of physical fitness

- Chest compressions

And at least 2 of the following:

- Tachycardia

- Tachypnoe

- Central Cyanosis

- Throbbing / constriction in the lungs [16]

How quickly an altitude disease will progress depends on your general state of health, age, degree of hydration, medication you take, the speed with which you reach altitude. The most vulnerable are the climbers who have changed high altitude in a short period of time [10].

\section{Acetazolamide in prevention of altitude disease}

The best way to prevent altitude diseases is to alkaliate and slowly increase altitude. The use of pharmaceuticals can replace natural methods of prevention when there is no time for acclimatisation and when there is no possibility of rapid descent. Acetazolamide is 
pharmacological prophylaxis in acute mountain diseases [11, 22]. It is a strong inhibitor of carbonate anhydrase (CA).

Side effects of acetazolamide are most commonly described as mild, yet may occur:

- Paresthesias

- Taste disorder

- Polyuria

- Fatigue

- Gastrointestinal symptoms

- Serious side effects are rare [19].

Acetazolamide was used for the first time as an acclimatization aid in the 1960s [20]. Since then, studies have been carried out demonstrating the efficacy and recommended doses of the drug in the prevention of acute mountain disease.

Grissom and others in their studies indicate the efficacy of acetazolamide in the treatment of acute mountain disease. The study involved 12 climbers who climbed Mt. McKinley, where the peak is $6150 \mathrm{~m}$. They reported to the medical research station with symptoms of acute mountain disease. Climbers were divided into 2 groups. The first group received acetazolamide $250 \mathrm{mg}$ orally (6 climbers) while the second group received placebo (6 climbers). After 24 hours from the treatment with acetazolamide, it was found that 5 climbers from the group taking acetazolamide recovered, whereas the placebo subjects still suffered from acute mountain disease [9].

Patot and others. In their study, they assessed the efficacy of the recommended dose. They conducted a randomized double-blind, placebo-controlled study. The study involved 44 climbers who stayed for 24 hours at $4300 \mathrm{~m}$ without prior acclimatization. They were divided into two groups. The group receiving acetazolamide at a dose of $125 \mathrm{mg}$ per day bowed to 22 volunteers. The second group also consisted of 22 people, but took placebo. Prophylaxis was implemented 3 days before the start of climbing and on the first day at $4300 \mathrm{~m}$. In the study it was found that acetazolamide effectively influenced prophylaxis and reduced the frequency of AMS at $4300 \mathrm{~m}$. In the group taking acetazolamide, the incidence of AMS was $14 \%$ and in the placebo group - $45 \%$ [21].

The studies carried out in Nepal at the height of $4937 \mathrm{~m}$ are another evidence of positive action of acetazomide as a drug infecting acute mountain disease. A prospective, double-blind, randomized randomized placebo study was conducted. The study involved 154 people climbing the Lobuje peak. 74 people were given acetazolamide in a dose of $125 \mathrm{mg}$ twice daily and 80 people placebo. It was found that the group of people who were treated with acetazolamide had 
a lower incidence of AMS than the group taking placebo. In the placebo group, 20 (24.5\%) patients with AMS were registered, whereas in the group taking the drug 9 (12.2\%) [2].

Attempts were also made to compare the effects of acetazolamide with other drugs. In 2019, Burns et al. compared the effects of ibuprofen with acetazolamide in preventing acute mountain disease. The research was conducted on climbing White Mountain in California at $3810 \mathrm{~m}$. The volunteers were divided into two groups. One group took ibuprofen at a dose of $600 \mathrm{mg} 3$ times a day 4 hours before climbing, while the other group received acetazolamide at a dose of $125 \mathrm{mg} 2$ times a day, the night before climbing. 92 climbers, $49 \%$ (45 climbers) taking ibuprofen and 51\% (47 climbers) of acetazolamide completed the study. The incidence of acute mountain disease was $56.5 \%$, in the ibuprofen group it was $11 \%$ higher. Therefore, the investigators found that ibuprofen turned out to be a worse drug in prevention than acetazolamide in case of fast climbing [4].

Another drug subjected to comparison with acetazolamide was budesonide. For this purpose, a double-blind, randomized randomized study with plcebo was performed. Inhalational budesonide was compared with orally taken acetazolamide and placebo. The study involved 102 climbers, 32 of whom received budesonide, 35 acetazolamide and the rest received placebo. It was found that budesonide is not an effective method in the prevention of acute mountain disease [13].

The possibility of action of acetazolamide in combination with another drug was also tested. The study carried out in Kilimanjaro by Lesche et al. aimed to compare the use of tadafil and acetazolamide administered to 24 people with acetazolamide administered to 27 climbers as a preventive method in altitude disease (HAI). The group where tadafil was administered was found to have a nice lower HAI compared to the group taking acetazolamide alone [12].

The most commonly proposed dose of acetazolamide for prevention of acute mountain disease is $250 \mathrm{mg}$ per day.

In one study on the action of acetazolamide at various doses, the $250 \mathrm{mg}$ daily dose was found to be practically as effective in the prevention of acute mountain disease as higher doses $[18]$.

The studies conducted by Carlstea et al. They checked the effects of acetazolamide in the following doses: $125 \mathrm{mg}$ twice daily, $225 \mathrm{mg}$ twice daily and placebo twice daily. Studies were carried out on climbers in La Paz in Bolivia (3630 m). It was found that for people who are not mountaineers but tourists climbing below $3700 \mathrm{~m}$ the doses of acetazolamide should not be lower than $250 \mathrm{mg}$ twice daily. Such a dose was considered the most effective in prevention of AMS symptoms [5]. 
The recent studies have tried to determine whether the lower dose than the recommended one (125 $\mathrm{mg} 2$ ray per day) may also be effective in prevention of acute mountain disease. To this end, a prospective, double-blind, randomized randomized study of climbers at the Everest Base Camp in Nepal was carried out. In one group, the subjects received a dose of acetazolamide equal to $62.5 \mathrm{mg}$, and in the second group, used so far in the recommendations of $125 \mathrm{mg}$. It was found that the side effects of the drug were similar in both cases. The reduced dose helped to prevent acute mountain disease as well as the $125 \mathrm{mg}$ dose [15].

Moreover, an attempt was made to check whether acetazolamide is able to prevent lung oedema and cerebral oedema at high altitude. A randomized, double-blind, placebo-controlled study was carried out in the Everest region on 339 climbers who were only partially air-conditioned. The altitude at which they were examined was between $4250 \mathrm{~m}$ and $5000 \mathrm{~m}$. Echocardiography was used to check whether acetazolamide is capable of lowering the pulmonary artery pressure that causes HAPE. It was demonstrated that acetazolamide did not induce pulmonary artery pressure reduction. On this basis, it was concluded that it is not effective in prevention of pulmonary oedema and cerebral oedema at high altitudes [3].

\section{Conclusion}

Studies conducted to demonstrate the efficacy of acetazolamide in the prevention of acute mountain disease show that it is an effective drug. The recommended dose has been proven to be effective: $125 \mathrm{mg}$ twice daily. For this purpose, randomized double-blind placebo-controlled trials were conducted on different size groups of volunteers. The effect of acetazolamide was also compared to other drugs. It was proven that ibuprofen and budesonide are not as effective as acetazolamide. In one study a combination of tadafil with acetazolamide was performed, which turned out to have better AMS prevention effects than acetazolamide itself. However, there is no more evidence. Worldwide studies have also tried to answer the question whether another dose of acetazolamide will be as effective as $250 \mathrm{mg}$ per day. No differences were found in studies where subjects were given smaller doses during climbing. However, there are no more studies to support this thesis. Acetazolamide is a good pharmacological solution when there is no possibility of acclimatization during climbing. Currently, the recommended dose (250 mg per day) is effective in the prevention of acute alpine disease. However, Acetazolamide does not work as a drug to prevent altitude cerebral oedema and altitude pulmonary oedema. 


\section{References}

1. $\quad$ Barry PW., Pollard AJ.: Altitude illness, BMJ. 2003; 326(7395): 915-919.

2. Basnyat B., Gertsche JH., Johnson EW., Castro-Marin F., Inoue Y., Yeh Clement.: Efficacy of Low-Dose Acetazolamide (125 Mg BID) for the Prophylaxis of Acute Mountain Sickness: A Prospective, Double-Blind, Randomized, Placebo-Controlled Trial, High Alt Med Biol 2003; 4(1): 45-52.

3. Basnyat B., Hargove J., Holck PS., Srivastav S., Alekh K., Ghimire LV., Pandey K., Griffitsh A., Shankar R., Kaul K., Paudyal A., Stasiuk D., Basnyat R., Davis Ch., Southard A., Robinson C., Shandley T., Johnson DW., Zafren K., Williams S., Weiss EA., Farrar JJ., Swenson ER.: Acetazolamide fails to decrease pulmonary artery pressure at high altitude in partially acclimatized humans, High Alt Med Biol 2008; 9(3): 209-216.

4. Burns P., Lipman GS., Warner K., Jurkiewicz C., Phillips C., Sanders L., Soto M., Hackett P.: Altitude sickness prevention with ibuprofen relative to acetazolamide, Am J Med 2019; 132 (2): 247-251.

5. Carlsten Ch., Swenson ER., Stephen R.: A Dose-Response Study of Acetazolamide for Acute Mountain Sickness Prophylaxis in Vacationing Tourists at 12,000 Feet (3630 M), High Alt Med Biol 2004; 5(1): 33-39.

6. Dehnert C., Bärtsch P.: Acute Mountain Sickness and High-Altitude Cerebral Edema, Ther Umsch. 2017; 74(10): 535-541.

7. Derby R., deWeber K.: The athlete and high altitude, Curr Sports Med Rep 2010; 9(2): 79-85.

8. Dumont L, Mardirosoff C, Tramer MR.: Efficacy and harm of pharmacological prevention of acute mountain sickness: quantitative systemic review. BMJ. 2000;321:267-272.

9. Grissom CK., Roach RC., Sarnquist FH., Hackett PH.: Acetazolamide in the treatment of acute mountain sickness: clinical efficacy and effect on gas exchange, Ann Intern Med 1992; 116(6): 461-465.

10. Hartman-Ksycińska A., Kluz-Zawadzka J., Lewandowki B.: Choroba wysokościowa, Przegl Epidemiol 2016; 70(3): 490-499.

11. Joyce KE., Lucas SJE., Imray CHE., Balanos GM., Wright AD.: Advances in the Available Non-Biological Pharmacotherapy Prevention and Treatment of Acute Mountain Sickness and High Altitude Cerebral and Pulmonary Oedema, Expert Opin Pharmacother 2018; 19(17): 1891-1902. 
12. Leshem E., Caine Y., Rosenber E., Maaravi Y., Hermesh H., Schwartz E.: Tadafil and Acetazolamide versus acetazolamide for the prevention of serve high-altitude illness, $\mathbf{J}$ Travel Med. 2012; 19(5): 308-10.

13. Lipman GS., Pomeranz D., Burns P., Phillips C., Cheffers M., Evans K., Jurkiewicz C., Juul N., Hackett P.: Budesonide versus acetazolamide for prevention of acute mountain sickness, Am J Med. 2018; 131: 200.e9-200.e16 .

14. Maggiorni M.: Mountaineering and altitude sickness, Ther Umsch 2001; 58(6): 387-393.

15. Mclntosh SE., Hemphill M., McDevitt M., Gurung T., Ghale M., Knott JR., Thapa GB., Basnyat B., Dow J., Weber D., Grissom CK.: Reduced acetazolamide dosing in countering altitude illness: a comparison of $62.5 \mathrm{vs} 125 \mathrm{mg}$ (the RADICAL trial), Wilderness Envirom Med 2019; 30(1): 12-21.

16. Piechocki J., Janus T.: Selected health-threatening and life-threatening conditions related to high altitude exposure, Anesthesiology Resuce Medicine Science Practice 2016; 10: 103-111.

17. Przybyłowski T., Zieliński J.: Problemy medyczne związane z przebywaniem na dużej wysokości, Pneumonol. Alergol. Pol 2004; 72: 134-138.

18. Ritchie ND., Baggott AV., Todd A.: Acetazolamide for the prevention of acute mountain sickness-a systematic review and meta-analysis, J Travel Med 2012; 19(5): 298-307.

19. Schmickl ChN., Owens RL., Orr JE., Edwards BA., Malhotra A.: Side effects of acetazolamide: a systematic review and meta-analysis assessing overall risk and dose dependence, BMJ Open Respir Res 2020; 7(1): e000557.

20. Swenson E.: Pharmacology of acute mountain sickness: old drugs and newer thinking, J Appl Physiol (1985) 2016; 120(2): 204-215.

21. Tissot van Patot M., Leadbetter H., Keyes LE., Maakeastad KM., Olson S., Hackett HP.: Prophylactic low-dose acetazolamid reduces the incidence and severity of acute mountain sickness, High Alt Med Biol. 2008; 9(4): 289-293.

22. Wright AD., Brearey SP., Imray Che.: High Hopes at High Altitudes: Pharmacotherapy for Acute Mountain Sickness and High-Altitude Cerebral and Pulmonary Oedema, Expert Opin Pharmacother 2008; 9(1): 119-127.

23. Zawadzki A.: Medycyna ratunkowa i katastrof. Warszawa PZWL 2006, 123-125. 